\title{
Experimental study on duodenal food passage vs. non-duodenal food passage reconstruction
}

\author{
Mari Morita, Kyoji Ogoshi \\ Department of Gastroenterological Surgery, Tokai University
}

\begin{abstract}
Background:

Our clinical data accumulated during 30 years of clinical practice at the Department of Gastroenterological Surgery, Tokai University, indicated the effectiveness of the Billroth 1 procedure that preserve duodenal food passage, as well as its suppressive effect on hepatic metastasis. Here, the effectiveness of food passage through the duodenum is examined via experiments using $\mathrm{BALB} / \mathrm{c}$ mice.

Methods:

In the first phase, gastrojejunostomy was performed using BALB/c mice. In the second phase, by duodenum ligation or not, the non-duodenal passage and duodenal passage models, respectively, were created. Transplantable colon 26 was transplanted into the spleen, and the number of hepatic metastases was examined. At the same time, Kupffer cells, NK cells, Th1 cytokines, and Th2 cytokines such as IL-12, INF $\gamma$, and IL-4 were measured in the sham operation mice.

Results:

(1) Hepatic metastasis was observed in 9 of 25 mice (36.0\%) and 18 of 26 mice (69.2\%) in the duodenum passage model and non-duodenum passage model, respectively $(\mathrm{p}=0.017, \mathrm{RR}=4.000,95 \% \mathrm{CI}, 1.246-12.842)$, and the average numbers of metastasis were 0.76 and 3.12 , respectively $(\mathrm{p}=0.077)$.

(2) No significant differences were observed in the number of Kupffer cells and NK activity, and the production of Th1 cytokines and Th2 cytokines between the two groups.

Conclusion:

It was considered that in non-duodenum passage reconstructive surgery that produced bacterial translocation due to the existence of a blind loop may have induced cytokine production, causing the activation of NK cells and leading eventually to hepatic metastasis.
\end{abstract}

Key Words: Duodenal food passage, non-duodenal food passage, mouse experimental study, NK cell, Kupffer cell

(Received August 29, 2011; Accepted September 22, 2011)

\section{Introduction}

Since Billroth performed the first successful surgery for cancer of the stomach in 1881, using the Billroth 1 procedure $^{1)}$, various reconstructive surgery have been reported. In 1884, Billroth reported the Billroth 2 procedure. Since then, various modified procedures of Billroth 1 and 2 have been reported as a basic reconstructive procedure for the stomach cancer. In 1897, Schlatter succeeded in a total gastrectomy ${ }^{2)}$, which became eager for discussion among surgeons worldwide regarding whether or not food should be allowed to pass through the duodenum $^{3)}$.

The procedures with all possibilities of thinking for surgical reconstruction after gastrectomy have been performed. These procedures can basically be classified as either: (1) physiological reconstructive surgery,

Correspondence to: Mari Morita, Department of Gastroenterological Surgery, Tokai University, 143 Shimokasuya, Isehara, Kanagawa 259-1193, Japan. TEL: +81463-96-6163; FAX: + 81-463-96-4120, e-male: marimorita@msn.com which allows food to pass through the duodenum, or (2) non-physiological reconstructive surgery, which does not allow food to pass through the duodenum. In general, the procedures are discussed in terms of factors such as difficulty of the operation (tension at the site of anastomosis) and postoperative complications (sutural insufficiency, postoperative pneumonia, bile reflux, and frequency of residual gastritis). In the West, unlike in Japan, postoperative complications such as sutural insufficiency and postoperative pneumonia are frequently observed due to differences in the physical constitution of the patients. Therefore, the Billroth 2 procedure is often selected in the West. On the other hand, the Billroth 1 procedure, which is a physiological reconstructive surgery procedure with duodenal food passage, is preferred in Japan. Various clinical parameters including QOL have been compared between the Billroth 1 and Billroth 2 procedures. However, the results of these comparisons are considered to be controversial because of their poor reproducibility and evidence. In addition, their prognosis is also controversial as some reports suggested a possible 
difference in prognosis between Billroth 1 and 2, while others do not ${ }^{4,5)}$. However, the analysis of our long-term follow up data has revealed that the prognosis was better for physiological reconstructive surgery with duodenal food passage than for non-physiological reconstructive surgery without duodenal food passage (unpublished data). In this study, we present an experimental examination of the significance of duodenal food passage using mice.

\section{Experimental Materials and Methods}

\section{Animals}

BALB/c mice, female, 5-8 weeks-old, were purchased from CLEA Japan, Inc.

\section{Cancer cell strains}

The cancer cell strain used was colon26 (a colon cancer cell line of BALB/c), which has been delivered at Tokai University School of Medicine, was used. The suspension of colon26 was prepared as follows. Colon26, which is subcultured in the fascia lata of mice, was removed, finely chopped, and then dissolved in a solution of $100 \mathrm{mg}$ of $0.2 \%$ collagenase in $50 \mathrm{~mL}$ of RPMI-1640 with $10 \%$ of FCS by stirring for 30 minutes at $37^{\circ} \mathrm{C}$. The resulting solution was filtered and the filtrate was washed by 5 minutes of centrifugation at 1,000 rpm. The precipitated cells were suspended in RPMI-1640 culture medium with $10 \%$ FCS to prepare a cell suspension with a concentration of $1 \times 10^{4}$ cells $/ \mathrm{mL}$.

\section{Preparation of the duodenal passage model and non-duodenal passage model}

BALB/c mice were starved for 4 hours or longer with water available ad libitum, before general anesthesia. Intraperitoneal injection of a mixture of $10 \mathrm{mg} / \mathrm{kg}$ of ketamine and $100 \mathrm{mg} / \mathrm{kg}$ of xylazine for median laparotomy. The stomach was identified and the omentum was pulled away to the cranial side. Approximately $3 \mathrm{~mm}$ of incisions were made in the greater curvature of the glandular stomach and jejunum to create anastomotic sites, which were anastomosed in a side-to-side fashion with 7-0 silk suture. When anastomosis was completed, the inside of the abdominal cavity was washed with $2 \mathrm{~mL}$ of saline solution, before the laparotomy incisions were closed in a single layer using 3-0 silk suture. After completion of the surgery, $1 \mathrm{~mL}$ of saline solution was injected subcutaneously in the back. The mice were given water and Ensure Liquid ad libitum from the day of the operation and 1 day after the operation, respectively. The mice were given standard solid food from 2 days after the surgery. After the gastrojejunostomy the mice were monitored for 2 weeks. The surviving mice underwent laparotomy under general anesthesia one more time, and the duodenum of selected mice were ligated using 4-0 silk suture to create the non-duodenal passage group (duodenum ligation group: DL). The duodenum passage group (gastrojejunostomy group: (GJ) consisted of those mice whose duodenums were not ligated after the second laparotomy. As a preparation for transcutaneous transplantation of cancer cells into the spleen at 2 weeks after the second laparotomy, a subcutaneous pocket was made during the second laparotomy by constructing the stoma in the left peritoneum, and the spleen was pulled out to the subcutaneous area along with the gastrosplenic ligament and placed into the pocket ${ }^{6)}$. This operation was conducted in both groups.

\section{Isolation and measurement of Kupffer cells}

After euthanization of the mice without inoculation of cancer cells, the liver was perfused from inferior vena cava with $10 \%$ FCS added RPMI-1640 culture medium, and incuvated in RPMI-1640 culture medium with 100 mg of $0.2 \%$ collagenase and $10 \%$ FSC for 30 minutes at $37^{\circ} \mathrm{C}$. The liver was finely crushed using the tip of a pipette and on a slide glasses. The suspension was pipettted up and down and the obtained supernatant was mixed with PBS with $1 \mathrm{~m}$ Eq EDTA. This mixture suspension was centrifuged for 10 minutes at $1,000 \mathrm{rpm}$, and the precipitate was centrifuged again for 5 minutes at 2,000 rpm. Density gradient centrifugation was carried out for this precipitate using $70 \%, 55 \%$, and $40 \%$ of percoll solution and PBS. Kupffer cells accumulating in the $55 \%$ phase and at the boundary between the $55 \%$ and $40 \%$ phases were collected. The cells were stained with trypan blue and measured using a cytometer. The solution of the obtained Kupffer cells with a concentration of $5 \times 10^{5}$ cells $/ \mathrm{mL}$ was incubated at $37^{\circ} \mathrm{C}$ in $5 \% \mathrm{CO}_{2}$ for 1 hour, 2 hours, 4 hours, and 24 hours. The culture supernatant was collected and the level of cytokines was measured. A mouse immunoassay kit from BIOSOURCE (USA) was used to measure the cytokines.

\section{Isolation and measurement of NK cells}

The mice without transplantation of cancer cells were sacrificed by cervical traction, and the liver and spleen were removed after laparotomy. The liver and spleen were finely chopped in RPMI-1640 culture medium with $10 \%$ FCS, crushed on a slide glass, and filtered under pressure through a metal mesh with a pore size of 0.2 $\mathrm{mm}$, using the inner cylinder of a syringe. The obtained solution was centrifuged for 10 minutes at 2,000 rpm. The precipitate was mixed with $33 \%$ Percoll solution and centrifuged for 18 minutes at 1,800 rpm. One (1) $\mathrm{mL}$ of lysis buffer was added to the precipitated RBC and the mixture was left to stand for lysis. The lysate solution was then filtered using a nylon mesh. RPMI-1640 medium with $10 \%$ FCS was added to the filtrate, and the mixture was centrifuged for 5 minutes at 1,500 rpm. The number of cells in the resulting precipitate was then 
counted. The radioactivity of ${ }^{51} \mathrm{Cr}$ released after 4 hours in the mixed culture, using E/T ratios of $25 \%$ (ET25), $50 \%$ (ET50), and 100\% (ET100) was measured to calculate the NK activity using the following formula.

$$
\begin{aligned}
\% \text { lysis }= & (\text { experimental count- spontaneous count }) / \\
& (\text { total count- spontaneous count }) \times 100
\end{aligned}
$$

\section{Experimental schedule}

Exp. 1

$1 \times 10^{4}$ cells of colon 26 were transcutaneous inoculated into the spleen of each mouse of the duodenum passage model (GJ) and non-duodenum passage model (DL). The mice were euthanized 4 weeks after the transplantation. The liver weight was measured and the number of hepatic metastases was macroscopically counted.

\section{Exp. 2}

The mice without transplantation of cancer cells receiving duodenum passage or non-duodenum passage reconstruction were euthanized 28 days after the surgery. The number of hepatic Kupffer cells and the level of cytokines such as IL-12, INF $\gamma$, and IL-4 were measured.

\section{Exp. 3}

The NK cell activities in the liver and spleen were measured and compared between the duodenum passage group (GJ) and non-duodenum passage group (DL) in mice without transplantation of cancer cells.

YAC-1 cells were labeled with ${ }^{51} \mathrm{Cr}$, and they were used as the target cells, while NK cells isolated from the liver and the spleen of the duodenum passage model (GJ) and non-duodenum passage model (DL) were used as the effector cells.

The above-mentioned experiment was designed in accordance with UKCCR guidelines for the welfare of animals in experimental neoplasia ${ }^{7)}$ and the Tokai University code of ethics for animal experiments.

\section{Statistical Analysis}

All statistical analyses were carried out using SPSS software, version 18 (SPSS Inc., Chicago, IL, USA). Mean values were compared using Student's-test with standard error (SE). A $\chi 2$-square test was used to compare the prevalence of Data with relative risk and $95 \%$ CI. Results were considered both side significant when $\mathrm{p}$ $<0.05$.

\section{Results}

\section{Hepatic metastasis model (Experiment 1)}

Hepatic metastasis was observed in 9 of 25 animals $(36.0 \%)$ and 18 of 26 animals $(69.2 \%)$ in the duodenum passage model (GJ) and non-duodenum passage model (DL), respectively ( $\mathrm{p}=0.017, \mathrm{RR}=4.000,95 \% \mathrm{CI}, 1.246$ -
12.842). The average number of macroscopic hepatic metastasis was $0.76 \pm 0.37$ and $3.12 \pm 1.23$ in the duodenum passage model (GJ) and non-duodenum passage model (DL), respectively ( $\mathrm{p}=0.077$ ) (Fig. 1).

2. The number of Kupffer cells and cytokine productivity (Experiment 2) and the number of NK cells (Experiment 3) in mice without transplantation of cancer cells.

\section{(1) Kupffer cells}

The number of Kupffer cells is shown in Fig. 2. No significant difference was observed between these groups.

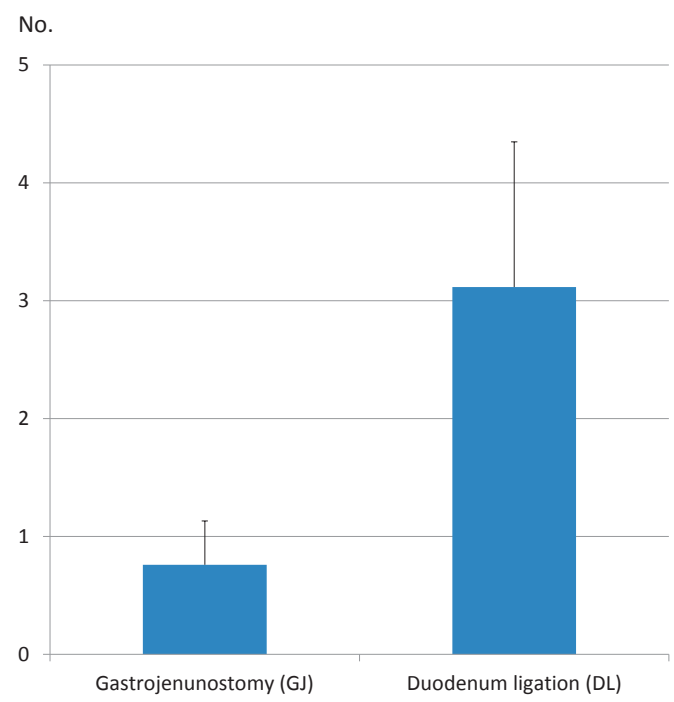

Fig. 1 The number of macroscopic hepatic metastasis in duodenal passage model mice (gastrojejunostomy: GJ), and non-duodenal passage model mice (Duodenum ligation: DL). Error bars, SE.

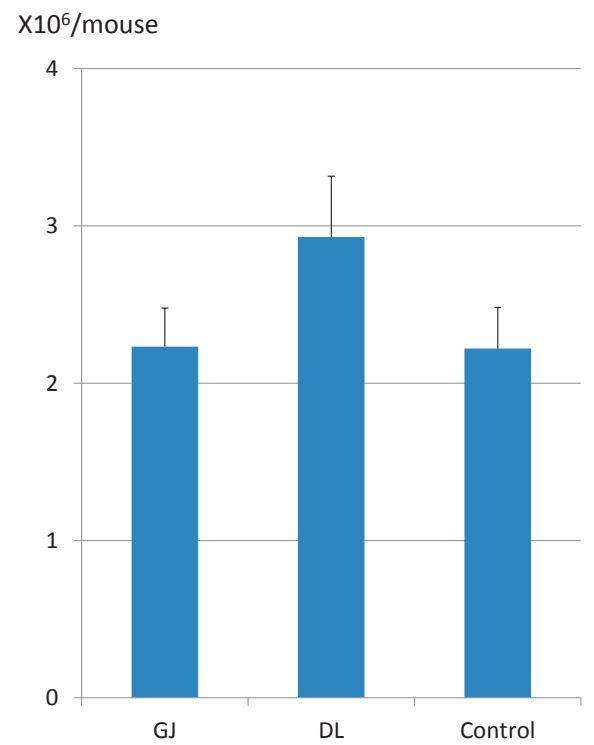

Fig. 2 Kupper cell in duodenal passage model mice (GJ), non-duodenal passage model mice (DL) and control. Error bars, SE. 


\section{(2) Cytokines}

The changes over the cultivation time in the levels of IL4, IL12, and INF $y$ are shown in Fig. 3. The levels in the surgery group were significantly lower than those in the control group. The levels in the duodenum passage group (GJ) at 24 hours were higher than those in the control group, while no significant difference was observed between the duodenum passage group (GJ) and the nonduodenum passage group (DL).

\section{(3) NK cells}

The number of NK cells is shown in Fig. 4. The number of NK cells in the spleen was significantly lower in the non-duodenum passage group (DL) than in the control group. The number of NK cells in the liver of the non-duodenum passage group (DL) was significantly higher than those in the duodenum passage group (GJ) and the control group.

\section{(4) NK activity:}

The NK activity in the spleen in the non-duodenum passage group (DL) was significantly higher than that in the control group, at all ET ratios (ET25, ET50, and ET100), while no significant difference was observed between the duodenum passage group (GJ) and the control

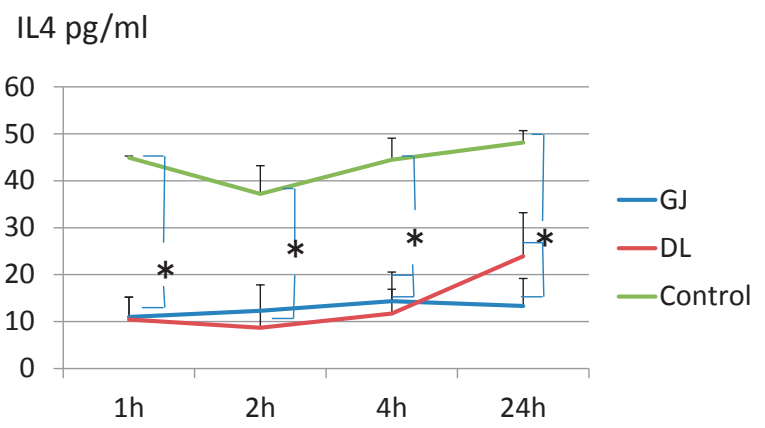

IL12 pg/ml

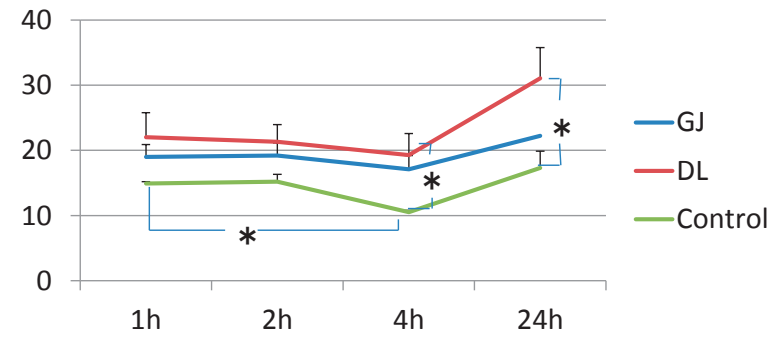

INF $\gamma \mathrm{pg} / \mathrm{ml}$

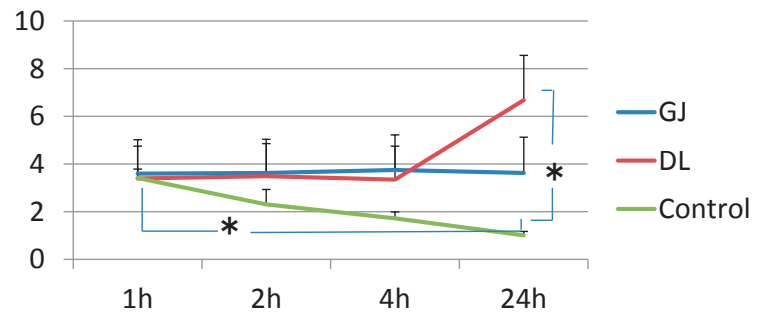

Fig. 3 IL4, IL12, and INF $y$ induodenal passage model mice (GJ), non-duodenal passage model mice (DL) and control. Error bars, SE. ${ }^{*}, \mathrm{p}<0.05$.

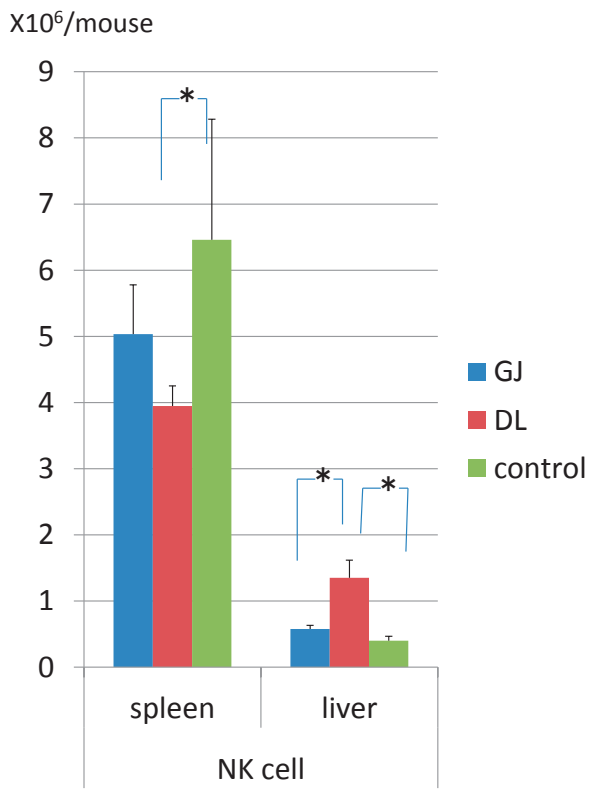

Fig. 4 Hepatic and spleen NK cell in duodenal passage model mice (GJ), non-duodenal passage model mice (DL) and control. Error bars, SE. ${ }^{*}, p<0.05$.

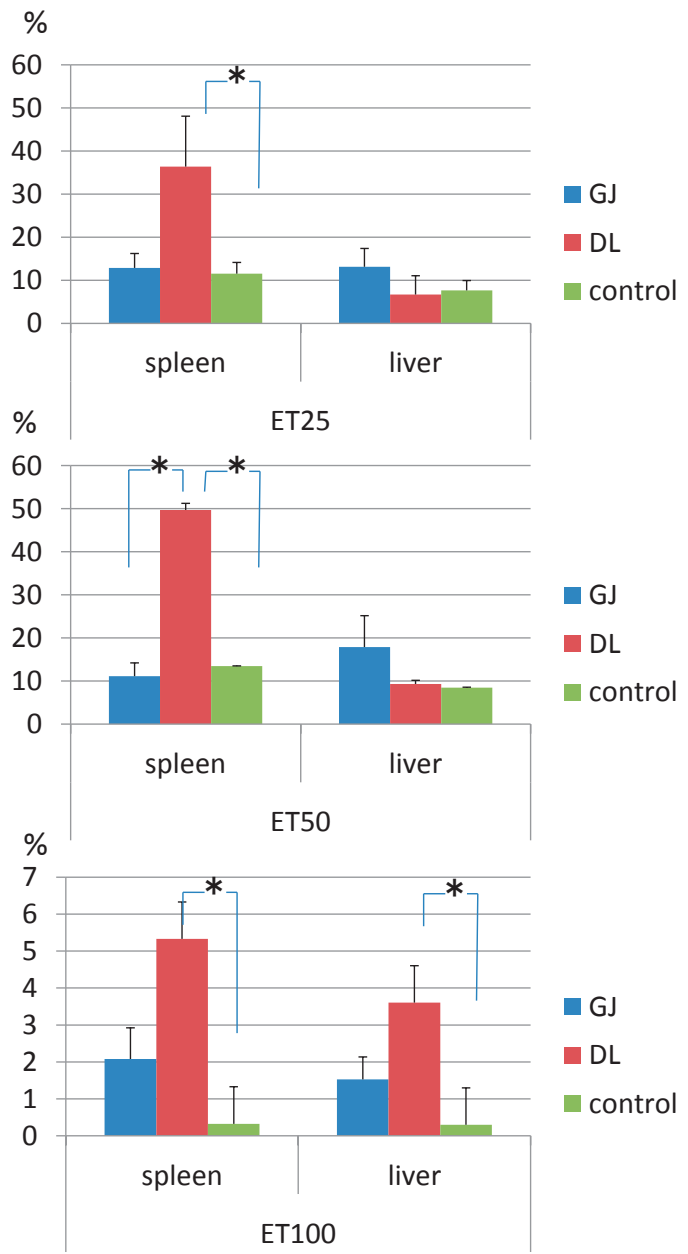

Fig. 5 NK cell activity $\left({ }^{51} \mathrm{Cr}\right.$ release in hepatic and spleen NK cell) according to $E / T$ ratio in duodenal passage model mice (GJ), non-duodenal passage model mice (DL) and control. Error bars, SE. ${ }^{*}, p<0.05$. 
group. A significant difference was observed between the non-duodenum passage group (DL) and the duodenum passage group (GJ) at ET50. Regarding the NK activity in the liver, a significant difference was observed between the duodenum passage group (GJ) and the control group at ET100 (Fig. 5).

\section{Discussion}

No report has been published regarding an experimental examination of the advantages and disadvantages of the Billroth 1 procedure (duodenum passage procedure) and the Billroth 2 procedure (non-duodenum passage procedure) using mice, since it is technically difficult to perform laparotomy and gastrojejunostomy on mice. The preparation of the gastrectomy model in this study was extremely difficult, due to these technical challenges. The results of this experimental examination have revealed that hepatic metastasis frequently occurs in the non-duodenum procedure. These results agree with our clinical data; however, the mechanism for this metastasis has not been made clear. In this study on normal model mice without transplantation of cancer cells, the number of NK cells in the spleen in the non-duodenum passage group was significantly lower than that in the control group, and the number of NK cells in the liver in the non-duodenum passage group was significantly higher than those in the duodenum passage group and the control group. The NK activity in the liver in the nonduodenum passage group was also significantly higher than those in the duodenum passage group and the control group. This experiment showed that the levels of IL4, IL-12, and INF $y$ in the non-duodenum passage group were significantly higher than those in the control group, suggesting that the preparation of the non-duodenum passage group stimulated the cytokines, resulting in the activation of NK cells and NK activity in the liver and spleen, which eventually led to hepatic metastasis.

In many of the reports examining the mechanism of hepatic metastasis of various tumors, the experiments are conducted using rats. In relation to the mechanism of regulation of metastasis, hepatic Kupffer cells and NK cells present in the liver sinusoidal lumen are of particular interest. Higuchi, et al. has reported that Kupffer cells, which were isolated from the liver and cultured, induce apoptosis and necrosis of cancer cells in rats. It was also reported that Kupffer cells exert an antineoplastic effect by producing cytokines such as TNF- $\alpha$, as well as releasing NO and active oxygen ${ }^{8)}$. Maarten, et al. suggested that coordinated antigen presentation and phagocytosis by Kupffer cells and NK cells play an important role in the local control of hepatic metastasis, based on the fact that Kupffer cells and NK cells exhibit phagocytic activity and antigen presentation against cancer cells within 24 hours of invasion of cancer cells into the liver ${ }^{9)}$. It has also been reported that phagocytes, including Kupffer cells, are recruited around the microscopically visible metastatic lesion formed 3 days after the invasion of cancer cells into the liver, and that the number of Kupffer cells peaked at Day $14^{10)}$. Kupffer cells were reported to induce the apoptosis of cancer cells by the Fas/Fas ligand system ${ }^{11)}$. It was also reported that the inhibition of Kupffer cells and NK cells increases the rate of hepatic metastasis in these experimental systems ${ }^{12)}$. Regarding the effect of NK activity, there is a report of an experiment using rats, stating that the inhibitory effect on tumor metastasis of intraportally administered OK-432 is higher than that of intravenously administered OK-432, based on a comparison of the changes in NK cells and the inhibitory effect on hepatic metastasis ${ }^{13)}$. There is also a report which suggests the possibility that NK cells may acquire a specific sensitizing action to inhibit hepatic metastasis, since hepatic metastasis was inhibited and NK activity was increased in the mice to which tumor cells were intradermally inoculated 14 days before the inoculation of tumor cells to the portal area ${ }^{14)}$. In the report of the experimental model using C57BL/6 by Nakura, et al. in which morphological changes in the liver sinusoid after the infusion of Colon38 into the superior mesenteric vein was examined, it is stated that the adhesion of tumor cells and lymphoid cells, as well as the destruction of tumor cells by lymphoid cells were observed until 3 days after the inoculation of Colon38, and that hepatic micrometastasis was observed using scanning electron microscopy from 4 days after the inoculation. It was also reported that hepatic metastasis was accelerated by the inhibition of NK cell activity by BRM, while hepatic metastasis was inhibited by the activation of NK cells ${ }^{15)}$.

In the liver of rats to which IL-12 had been administered, NK cells were considered to play a defensive role at the early stage of hepatic metastasis by being trapped in Kupffer cells activated by endogenous INF- $\gamma$, which increased in the sinusoid $^{16)}$. Vermijlen, et al. predicted that the activation of hepatic NK cells inhibits hepatic metastasis $^{17)}$. The above-mentioned reports, which state that NK cells inhibit hepatic metastasis, contradict the data we have obtained in this study.

On the other hand, it has been reported that, under specific conditions such as burns and total parenteral nutrition, Kupffer cells cause hepatocellular damage. Morikawa, et al. suggested the possibility that a hepatic disorder caused by bile congestion in the total parenteral nutrition experimental model using rats is attributable to the hepatic mitochondrial damage caused by activation of Kupffer cells and release of cytokines induced by bacterial translocation from the intestinal $\operatorname{tract}^{18)}$. Yamada, et al. reported that liver regeneration is suppressed by the activation of Kupffer cells attributable to bacterial translocation associated with portal vein congestion, which is 
caused by a combination of hepatoportal and total hepatic ischemia, in an experiment using rats ${ }^{19)}$. Furuichi, et al. indicated that ischemia induces tumor vessels, based on the fact that the hepatic metastasis of colon cancer cells transplanted into the spleens of mice is accelerated by creating a temporary ischemic state in the liver ${ }^{20)}$. According to the report by Guler, et al., although mice are originally Th2-dominant organisms, neonatal thymectomy of BALB/c tips the Th1/Th2 balance in favor of Th1, resulting in the activation of both Th1 and Th2 immune reactions ${ }^{21)}$. In addition, when these $\mathrm{BALB} / \mathrm{c}$ mice were infected with Helicobacter pylori after the thymectomy, a MALT lymphoma-like lesion developed while it was not observed in mice without thymectomy. Therefore, it is considered that specific cytokines produced by the host play an important role in the induction and execution of immune reactions, and determine the sensitivity and resistance to infection ${ }^{20,22)}$. Based on the results of this study using normal mice, combined with the above-mentioned reports, it is considered that in non-physiological reconstructive surgery using the nonduodenum passage procedure in which a blind loop is created, the activation of NK cells caused by bacterial translocation may induce the hepatic metastasis. It is necessary to examine the timing of the colonization of the tumor cells in the liver and the timing of the activation and the killing of NK cells in the tumor-bearing state. However, no report has been published which examined cytokines and bacterial translocation.

\section{Conclusion}

As expected from the clinical data, the number of cases of hepatic metastasis was significantly higher in the non-duodenum passage group, compared to the duodenum passage group. It is considered, from the examination of the number of Kupffer cells and NK cells, as well as cytokine productivity, that in the non-physiological reconstructive surgery using the non-duodenum passage procedure, in which a blind loop is created, the activation of NK cells caused by bacterial translocation and stimulation of cytokines may induce hepatic metastasis. It is considered in the future necessary to examine the changes in immunological markers, especially NK cells, using tumor-bearing mice.

\section{Acknowledgments}

We wish to thank Prof. Kyoji Ogoshi for his continuous support. We also wish to thank Akemi Kamijo of the Teaching and Research Support Center, Tokai University School of Medicine, and Izumi Tsuchiya of the Department of Surgery, Tokai University School of Medicine, for their cooperation in conducting this experiment.

\section{Reference}

1) Billroth, T. (1881) Offense Schreiben an Herrn Dr. L.Wittelshofer. Wienr Medizinische Wochenschrift Nr. 6:161-165.
2) Schlatter, C. (1897) Ueber Ernahrung und Verdauung nach vollstandiger Entfernung des Magens-Oesophagoenterostomie-beim Menschen. Beitr Klin Chir 19:757-776.

3) Piessen, G., Triboulet, J-P., Mariette, C. Reconstruction after gastrectomy (2010) Which technique is best? Journal of visceral surgery. 147:e273-83.

4) Chareton, B., Landen, S., Manganas, D., Meunier, B., Launois, B. (1996) Prospective randomized trial comparing Billroth I and Billroth II procedures for carcinoma of the gastric antrum. Journal of the American College of Surgeons. 183:190-194.

5) Montesani, C., D’Amato, A., Santella, S., Pronio, A., Giovanni, C., Cristaldi, M. (2002) Billroth I versus Billroth II versus Rouxen-Y after subtotal gastrectomy. Prospective randomized study. Hepatogastroenterology 49:1469-1473.

6) Fujii, M., Konn, Y., Yamanaka, Y., Miyagishima, K., Morita, T., Sasaki, M., Ono, K. (1986) Intrasplenic SerialInjection of Lipopolysaccharide:Induction of Anti-Tumor Substance in Ratty Portal Vein. Nihon Geka Gakkai Zasshi :87(2), 237.(in Japanese with English abstract)

7) Workman, P., Balmain, A., Hickman, J.A., McNally, N.J., Mitchison, N.A., Pierrepoint, C.G., Raymond, R., Rowlatt, C., Stephens, T.C., Wallace, J., Straughan, D.W., (1998) UKCCCR guidelines for the welfare of animals in experimental neoplasia. British Journal of Cancer. 58:109-113.

8) Higuchi, H., Ishii, H. (1997) Isolation and evaluation of Kupffer cell function: a review of studies by fluorographic techniques Kan Tan Sui. 35(6) : 865-871. (in Japanese with English abstract)

9) Timmes, M., Vekemans, K., Vermijlen, D., Asosingh, K., Wisse, P., Braet, F. (2004) Interactions Between Rat Colon Carcinoma Cells And Kupffer Cells During The Onset Of Hepatic Metastasis. International Journal of Cancer. 112:793-802.

10) Heuff, G., van der Ende, M.B., Boutkan, H., Prevoo,W., Bayon, L.G., Fleuren, G.J., Beelen, R.H., Meijer, S., Dijkstra, C.D. (1993) Macrophage populations in different stages of induced hepatic metastases in rats: an immunohistochemical analysis. Scandinavian Journal of Immunology. 38(1):10-16.

11) Okada, K., Wada, T., Ito, K., Takagi, Y., Aoki, T., Koyanagi, Y. (2000) Investigation of the Anti-Tumor activity of Hepatic Kupffer Cells for Control of Colon Cancer Micro-Metastasis to the Liver via the Fas/Fas ligand system. Journal of Tokyo Medical University. 61(4):329-335.

12) Rushfeldt, C., Sveinbjørnsson, B., Seljelid, R., Smedsrød, B. (1999) Early Events of Hepatic Metastasis Formation in Mice: Role of kupffer and NK-Cells in Natural and Interferon- $\gamma$-Stimulated Defense. Journal of Surgical Research. 82:209-215.

13) Akazaii, Y., Fuchimoto, S., Watanabe, T., Suzuki, N., Iwagaki, H., Orita, K. (1992) The Role of Liver-Associated Natural Killer Cells as The Host Immune System on The preventive Effect Against Hepatic metastases. BIOTHERAPY 6(5):756-758. (in Japanese with English abstract)

14) Shimotono, H. (1993) Inhibitation of Liver Metastasis by Sensitization :An Immunological Analysis. Journal of the Iwate Medical Association. 45(5):513-521.

15) Nagura, T. (1993) Morphological and Function Changes of Hepatic Sinusoidal Cells during Experimental Hepatic Metastasis of Colon Carcinoma. Dokkyo journal of medical sciences. 9(1):9-19. (in Japanese with English abstract)

16) Jinnai, H., Lee, Y., Okuno, K., Shigeoka, H., Hirai, N., Honda, S., Yasutomii, M. (1997) In Vivo Effects of Interleukin(IL-12) in Comparison With IL-2: Influence on Liver Lymphocyte. Biothrapy 11(3), 423-425. (in Japanese with English abstract)

17) Vermijlen, D., Luo, D., Froelich, C.J., Medema, J.P., Kummer, J.A., Willerms, E. Breat, F., Weiss, E. (2002) Hepatic Natural Killer Cells Exclusivery Kill Splenic/Blood Natural Killer-resistant Tumor Cells by the Perforin/Granzyme Pathway. Journal of Leukocyte Biology 72:668-676.

18) Nobuyuki Morikawa (2001) Pathogenesis of total parenteral nutrition associated cholestasis in the rat model; Dose discontinuous administration attenurate liver dysfunction? The Japanese Journal of parenteral and enteral Nutrition. 23(11):583-588. 
19) Masami Yamada, Shutaro Ozawa, Isamu Koyama (2004) The Pringle maneuver Accelerates Liver Metastasis by Over-Expression of Tumor Vasculature in a Murine Model of Colon Cancer. Nihon Geka Rengo Kaishi 29(1):31-33.

20) Furuchi, K., Usami, M., Ohyanagi, H., Saitoh, Y. (1993) Inhibitory effect of portal pooling, bacterial translocation, and Kupffer cell activation on hepatic regeneration after partial hepatectomy by repeated portal triad cross clamping in rats. Nippon Shokakibyo Gakkai Zasshi. 90(12):3006-3017. (in Japanese with English abstract)

21) Mehmet L. Guler, James D. Gorham, Chyi-Song Hsieh, Aaron J. Mackey, Robert G. Steen, William F. Dietrich, Kenneth M. Murphy. (1996) Genetic susceptibility to Leishmania: IL-12 responsiveness in Th1 cell development. Science 271:984-987.

22) Kazushige Uchida, Kazuichi Okazaki, Andras Debrecceni, Toshiki Nishi, Hirosi Iwano, Maki Inai, Suguru Uose, Hiroshi Nakase, Masaya Ohana, Chikashi Oshima, Yumi Matsushima, Chiharu Kawanami, Hiroshi Hiai, Toru Masuda, Tsutomu Chiba (2001) Analysis of Cytokines in the Early Development of Gastric Secondary Lymphoid Follicles in Hericobacter pyloriInfected BALB/c Mice with neonatal Thymectomy. Infection And Immunity: 6749-6754. 